\title{
A new partnership between the World Heart Federation and Nature Clinical Practice Cardiovascular Medicine
}

\author{
Valentin Fuster and Rachel Ashton
}

This Editorial marks the beginning of a new partnership between the World Heart Federation (WHF) and Nature Clinical Practice Cardiovascular Medicine. We are excited about the potential of this collaboration to bring cardiovascular medicine's best information to a global readership.

The WHF unites 186 cardiology societies and heart foundations in 100 countries, reaching patients, policy makers and the public the world over. The organization began as the International Society and Federation of Cardiology in 1978, after a merger of the International Society of Cardiology and the International Cardiology Federation, but has been operating as the WHF since 1998. The focus of the member organizations is prevention and control of heart disease and stroke in low-income and middle-income countries, with four main areas of activity: awareness building; advocacy; demonstration projects in which the in-country member organizations become involved in screening, treatment and prevention strategies and health promotion campaigns; and the sharing of science. Awareness-building activities range from raising the profile of the growing burden of cardiovascular disease through such programs as World Heart Day and Go Red for Women, to campaigning for policy change in the areas of tobacco control, healthy diets and physical activity. The WHF's advocacy efforts include working towards making cardiovascular disease and other chronic diseases part of the United Nations Millennium Development Goals. Demonstration projects are currently being run in Grenada, China, Russia and the Pacific Islands. Several ongoing, long-term projects are underway in collaboration with the WHO and the United Nations Educational, Scientific and Cultural Organization, and a number of other organizations. The sharing of science is achieved through world congress meetings, the WHF's original research journal Prevention and Control, and now through Nature Clinical Practice Cardiovascular Medicine. "...the WHF is reaching out to specialists in diabetes, hypertension, stroke, atherosclerosis, obesity, nephrology and other specialties...."

$V$ Fuster is the Editorin-Chief and R Ashton is the Editor of Nature Clinical Practice

Cardiovascular Medicine.

\section{Competing interests}

The authors declared they have no competing interests.

www.nature.com/clinicalpractice doi:10.1038/ncpcardio0544
The partnership between the WHF and Nature Clinical Practice Cardiovascular Medicine will help a global network of clinicians to access review articles and commentaries covering the latest science, practice and care developments in cardiovascular medicine. The natural outcome of this collaboration will be to increase the coverage of critical issues in patients' care in countries with developing economies, as well as of those affecting higher-income countries. Care and treatment guidelines must be understood within the context and constraints of individual health-care systems, in which economics play an overarching role, but there is also much to be learned across these boundaries. We feel that the content of Nature Clinical Practice Cardiovascular Medicine will complement the issues of population health and the role of the community already dealt with in Prevention and Control.

The reduction of the global burden of cardiovascular disease, particularly in low-income and middle-income countries, will involve the efforts of many people and organizations. Already, the WHF has a global network of member organizations and colleagues in public health and patient care. Still, however, the WHF is reaching out to specialists in diabetes, hypertension, stroke, atherosclerosis, obesity, nephrology and other specialities to improve the care of patients. The relationship with Nature Clinical Practice Cardiovascular Medicine and with the whole Nature Clinical Practice group can only facilitate this multidisciplinary outreach. Through this partnership we aim to help the WHF to stimulate the thinking of society members in cardiology and in other chronic diseases to improve care for many patients around the globe, and to provoke dialogue, comment and, most importantly, action.

The next World Congress of Cardiology meeting will be held in Barcelona, Spain, on September 2-6, 2006, simultaneously with the European Society of Cardiology Congress. 